\title{
Journal of IMAB
}

- Annual Proceeding (Scientific Papers) 2020

\author{
vol. 26, issue 1 \\ (January - March)
}

Publisher: 


\title{
Journal of IMAB - Annual Proceeding (Scientific Papers) Editorial Board \\ https://www.journal-imab-bg.org/en/editorial-board.htm
}

\author{
Editor-in-chief: \\ Prof. Dr Krassimir Metodiev \\ Deputy Editor-in-chief: \\ Assoc. prof. Dr George Kyuchukov \\ Editors:
}

Prof. Dr Angelina Kisselova-Yaneva (Responsible for part Oral\&Dental Medicine)

Prof. Dr Liana Gercheva (Responsible for part Medicine)

\author{
Associate Editors: \\ Prof. Dr Bogdan Petrunov, \\ Prof. Dr Dimitar K. Gospodinov \\ Dora Peychinska, \\ Dr Orlin Savov - Germany \\ Paula Lazarova,
}

\section{International Members:}

Prof. Dr Kurt G. Naber - Germany;

Prof. Dr Mario Di Gioacchino - Italy;

Prof. Dr Pio Conty - Italy;

Prof. Dr Dieter Falkenhagen - Austria;

Prof. Dr Ian Gould - UK;

Prof. Dr Bulent Berkarda - Turkey;

Prof. Dr Roman Kozlov - Russia;

Prof. Dr Dan Engelhard - Israel;

Prof. Dr Raphael Saginur - Canada;

\section{Part Medicine:}

Prof. Dr. Anelia Klissarova

Prof. Dr. Ara Garabed Kaprelyan

Prof. Dr. Dilyana Vicheva

Prof. Dr. Ekaterina Titianova

Assoc. prof. Dr. Galya Gancheva

Assoc. prof. Dr. Irina I. Ivanova

Assoc. prof. Dr. Ivelina Yordanova

Dr. Katharina Wassilew

Prof. Dr. Mariana Arnaoudova

Assoc. prof. Dr. Maya Danovska-

Mladenova

Prof. Dr. Snejina Vassileva

Prof. Dr. Snezhanka T. Tisheva-

Gospodinova

Prof. Dr. Sonja Genadieva-Stavric

Prof. Dr. Valentin K. Stoyanov

Prof. Dr. Zaharyi Krastev;
Part Oral\&Dental Medicine:

Assoc. prof. Dr Alessandro Leite Cavalcanti

Assoc. prof. Dr Ani Beltcheva,

Assoc. prof. Dr Assya Krasteva,

Assoc. prof. Dr Elica Deliverska,

Prof. Dr Elka Popova,

Prof. Dr Hristina Lalabonova,

Prof. Dr Hristina Mihailova,

Assoc. prof. Dr Ilyana Stoeva,

Assoc. prof. Dr Mariana Dimova,

Prof. Dr Metodi Abadjiev,

Prof. Dr Milena Peneva,

Assoc. prof. Dr Nikolay Yanev,

Prof. Dr Slave Naumovski

Prof. Dr Vladimir Panov,

Publisher\&Executive Director:

Gospodin Peytchinski

\section{Address for correspondence:}

\section{Editor-in-chief:}

Prof. Dr. Krassimir Metodiev

Headquarters of International Medical Association

Bulgaria (IMAB);

55, M. Drinov str., 9002 Varna, Bulgaria,

Tel.: +359/888 712407

E-mail: kr.metod@yahoo.com or

kr.metod@gmail.com

\section{Deputy Editor-in-chief:}

Assoc. prof. Dr. George Kyuchukov

Tel.: +359/888628 424

E-mail: kyuchger@abv.bg

\section{Publishing house:}

Peytchinski Publishing Ltd.

compl. Droujba bl. 116, ap. 41;

5806 Pleven, Bulgaria

E-mail: publisher.imab@gmail.com or

publisher_imab@abv.bg

Journal of IMAB - 2020 Jan-Mar;26(1):

ISSN: 1312-773X; DOI: https://dx.doi.org/10.5272/jimab.2020261 